Voix et Images

voixetimages

\title{
Bibliographie de Monique LaRue
}

\section{Richard Raymond}

Volume 28, numéro 2 (83), hiver 2003

Monique LaRue

URI : https://id.erudit.org/iderudit/006600ar

DOI : https://doi.org/10.7202/006600ar

Aller au sommaire du numéro

Éditeur(s)

Université du Québec à Montréal

ISSN

0318-9201 (imprimé)

1705-933X (numérique)

Découvrir la revue

Citer ce document

Raymond, R. (2003). Bibliographie de Monique LaRue. Voix et Images, 28(2),

109-122. https://doi.org/10.7202/006600ar d'utilisation que vous pouvez consulter en ligne.

https://apropos.erudit.org/fr/usagers/politique-dutilisation/ 


\section{Bibliographie de Monique LaRue ${ }^{1}$}

\section{Richard Raymond, Université du Québec à Montréal}

\section{OEUVRES}

\section{I.1. Romans}

La cohorte fictive, Montréal, L'étincelle, 1979, 121 p.; Montréal, Les Herbes rouges, 1986, $121 \mathrm{p}$.

Les faux fuyants, Montréal, Québec Amérique, coll. «Littérature d'Amérique» 1982 [1989], 201 p. ${ }^{2}$

Copies conformes, Montréal/Paris, Lacombe/Denoël, 1989, 189 p.; Montréal, Club Québec-Loisirs Inc., 1989, 189 p.; Montréal, Boréal, coll. «Boréal compact», 1998, 190 p.; True Copies, trad. Lucie Ranger, Montréal, Nuage éditions, 1996, 174 p.; Tanz der Doubletten, trad. allemande de Uli Aumüller, Hambourg, Rowohlt, coll. «Die Neue Frau » 1992, 186 p.

La démarche du crabe, Montréal, Boréal, 1995, 220 p.

La gloire de Cassiodore, Montréal, Boréal, 2002, 296 p.

\section{I.2. Nouvelles, extraits de romans en chantier $^{3}$}

«Mallarmé, côté cour», La Vie en rose, n 28, juillet-août 1985, p. 18-19.

«San Francisco-Montréal 》, Mœbius, n 29, été 1986, p. 71-80.

«La rivière Preston», XYZ. La revue de la nouvelle, n 11, automne 1987, p. 55.

«Leçon de choses», XYZ. La revue de la nouvelle, no 15, automne 1988, p. 25-29.

«Décembre», Agenda littéraire, Foire du livre de Lanaudière, 1988, non paginé.

«Paysage de nulle part et de jamais», Trois, Montréal, vol. V, nos 1-2, automne 1989, p. 13-17.

«Un soir, en 1967», Arcade, n² 25, automne 1992, p. 33-34.

«Mon enfant, ma sœur», Liberté, vol. XXXV, nos 4-5, août-octobre 1993, p. 176-185.

«Une photo » La Presse, 25 juin 1995, p. C-3.

1. Je tiens à souligner la généreuse contribution de Monique LaRue dans l'élaboration de cette bibliographie.

2. Un chapitre de ce roman a été traduit en anglais dans Between $C$ and D, vol. II, no 3 , hiver 1986, p. 22-25.

3. Publiés avant leur parution en version définitive, ces fragments provisoires ont été soit remaniés pour figurer dans un roman subséquent, soit complètement retirés de celui-ci. 


\section{I.3. Dramatiques à la radio}

L'enregistrement, diffusée le 12 octobre 1984 dans le cadre de l'émission Premières, à la première chaîne de Radio-Canada.

Babil, diffusée en septembre 1985 dans le cadre de l'émission Éloges: éloge de l'enfance, à la chaîne FM de Radio-Canada 4 .

L'orage, diffusée en novembre 1985 dans le cadre de l'émission Éloges: éloge de l'orage, à la chaîne FM de Radio-Canada.

Paysages de nulle part et de jamais, diffusée à l'été 1988 dans le cadre de l'émission Matière de rêve, à la chaîne FM de Radio-Canada.

\section{I.4. Collectifs}

«Babil», Plages, en collaboration, Montréal, Québec Amérique, 1986, p. 51-57.

«L'Aventure des ondes», André Carpentier (dir.), L'aventure, la mésaventure, Montréal, Les Quinze, 1987, p. 13-28.

«'exposition», Micheline LaFrance (dir.), Nouvelles de Montréal, Montréal, l'Hexagone, coll. «Typo » n 68, 1992, p. 116-126.

«Vas-y, Pétula», Récits de la fête, Montréal, Québec Amérique, coll. «Mains libres», 2000, p. 221-240.

«Thalès et le fleuve Méandre»(en collaboration avec Marc Séguin et Benoît Villeneuve), Dialogues dans l'espace-temps, textes littéraires sous la direction de Jean-François Poupart, Montréal, Renouveau pédagogique, 2002, p. 5.

\section{I.5. Essais}

Promenades littéraires dans Montréal (en collaboration avec Jean-François Chassay), Montréal, Québec Amérique, 1989, 274 p. ${ }^{5}$

L'arpenteur et le navigateur, Montréal, Fides/CÉTUQ (Centre d'études québécoises de l'Université de Montréal), coll. «Les grandes conférences»1996, 30 p.

4. Cette pièce radiophonique fut reprise dans Plages, voir section suivante (I.4 Collectifs).

5. Les chapitres de cet essai parurent également en page 3 du cahier $C$ de La Presse du samedi, de la mi-octobre 1989 à la fin janvier 1990 («L'univers de la taverne à Montréal», 15 octobre 1989; "Tramway ou métro, au terminus tout le monde descend... », 22 octobre 1989; «Le magasinage : une activité économique et... de loisir», 29 octobre 1989; "La Main exerce depuis toujours une fascination exceptionnelle», 5 novembre 1989; «Outremont, symbole des canadiens-français arrivés... rapidement», 12 novembre 1989; «La rue Sainte-Catherine, étriquée, abracadabrante et... vivante», 19 novembre 1989; «Dense en sédiments historiques, le Vieux-Montréal recèle le sens de Montréal», 26 novembre 1989; «Le Faubourg Saint-Denis, le foyer de l'imaginaire littéraire montréalais» 3 décembre 1989; «Le Plateau Mont-Royal et le charme baroque de ses escaliers» 10 décembre 1989; «La nuit, la vérité se dissimule derrière la réalité", 17 décembre 1989; «La présence du restaurant dans les romans》, 24 décembre 1989; «Ces lieux d'enfermement de la ville que sont les bagnes», 31 décembre 1989; «La deuxième guerre engendra de vives tensions à Montréal 》, 7 janvier 1990; «Le paradoxe de la double allégeance de Montréal, française et américaine» 14 janvier 1990; "Le clocher constitue à la fois un repère géographique et moral», 21 janvier 1990 ; «À Montréal comme ailleurs, les écrivains composent avec la mort», 28 janvier 1990). 


\section{I.6. Études et critiques littéraires}

«Sur l’hérö̈ne stéréotypée de la littérature enfantine» Le Devoir, 10 mai 1975, p. 21.

«Écrire pour les enfants: un délicat dosage de "pédagogie" et de "littérature"», Lurelu, vol. II, nº 4, hiver 1979, p. 4-6.

«Celles qui parlent», Spirale, n 9 , mai 1980, p. 7.

«La forme ardente de la fiction», Spirale, no 10, juin 1980, p. 1.

«Some American Best Sellers», Spirale, no 11, septembre 1980, p. 14.

«Le texte derviche», Spirale, no 13, novembre 1980, p. 4.

«Le culte auto-biographique», Spirale, n 15 , janvier 1981, p. 14.

«Une écriture liquide», Spirale, nº 17, mars 1981, p. 8.

«Les enjeux de l'écriture», Spirale, n 19 , mai 1981, p. 7.

«Une histoire de fous», Spirale, nº 20, juin 1981, p. 3.

«Une éthique du signe», Spirale, n 20, juin 1981, p. 7.

«Fausse représentation», Spirale, no 21, septembre 1981, p. 3.

«l faut écrire $1981 »$ Le Devoir, 28 novembre 1981, p. 26.

«L'impossible et la mère», Marguerite Duras à Montréal, textes réunis et présentés par Suzanne Lamy et André Roy, Montréal, Éditions Spirale, 1981; Paris/Montréal, Spirale/Solin, 1984, p. 85-90.

«Le sens de l'Histoire», Spirale, n 22, février 1982, p. 10.

«Le travail de la représentation», Spirale, n 22, février 1982, p. 10.

«Incursion chez les romanciers», Spirale, n 23, mars 1982, p. 3.

«Un voyage qui tourne court», Spirale, n 25 , mai 1982, p. 4.

«Au sujet du désir», Spirale, n 26, juin 1982, p. 14.

«Du système au fragment», Spirale, n 28 , octobre 1982, p. 4.

«La mère, aujourd'hui », La Nouvelle Barre du jour, n 116, septembre 1982, p. 51-55.

«Le travail d'écriture», Livraisons, 1983, p. 15.

«La drogue, le paradis et la vraie vie», Le Devoir, 12 février 1983, p. 18.

«Miroir de l’hédonisme», Le Devoir, 26 février 1983, p. 21.

«Edipe, quand tu nous tiens», Spirale, n 33, avril 1983, p. 12.

«Jeunes filles, sachez reconnaître le loup», Spirale, no 38, novembre 1983, p. 4.

«Une chevauchée planétaire», Spirale, no 39, décembre 1983, p. 12.

«Mon cœur soupire», Spirale, no 42, mars 1984, p. 3.

«La vérité est un couple», Spirale, n ${ }^{43}$, mai 1984, p. 9.

«En quête de la modernité », Possibles, vol. VIII, n 3, printemps 1984, p. 155-158.

«La disparition de 1984 », Le Devoir, 11 novembre 1984, p. 20.

«Espaces d'écriture», L'écrivain et l'espace: rencontre québécoise internationale des écrivains, Montréal, l'Hexagone, 1986, p. 81-84.

«Le dernier des puritains», Le Devoir, 21 mars 1987, p. D-4. 
«Les nouvellistes réfléchissent sur la nouvelle», Québec français, n 66, mai 1987, p. 6069.

«Québec, Haïti : même Floride», Le Devoir, 20 juin 1987, p. D-4.

«Quand le cliché concerne tout le monde: "To Be or Not to Be" en 1995», Le Devoir, 31 octobre 1987, p. D-4.

«Des mémoires apocryphes», Le Devoir, 12 décembre 1987, p. D-6.

«Entre France et Québec: des lieux, des liens, une voix », Voix et Images, n 37 , automne 1987, p. 42-45.

«'appel de l’Amérique», Le Devoir, 23 janvier 1988, p. D-4.

«Le grain de sable dans l'engrenage», Le Devoir, 19 mars 1988, p. D-4.

«Portrait de citadin », Le Devoir, 26 mars 1988, p. D-4.

«Effets à rebours», Le Devoir, 9 avril 1988, p. D-4.

«Moment immobile», Le Devoir, 16 avril 1988, p. D-8.

«Adultère chez les universitaires», Le Devoir, 23 avril 1988, p. D-4.

«La tentation de la vie» Le Devoir, 7 mai 1988, p. D-4.

«Les méandres d'une œuvre», Le Devoir, 21 mai 1988, p. D-4.

«Chair à fiction» Le Devoir, 28 mai 1988, p. D-4.

«Dieu, la Science et la Femme», Le Devoir, 11 juin 1988, p. D-4.

«'Âme américaine cernée peu à peu» Le Devoir, 22 octobre 1988, p. D-4.

«Des procédés d'écriture comme une promenade dans l'atelier d'un peintre», Le Devoir, 26 novembre 1988, p. D-4.

«L'enfermement des petites cultures», Le Devoir, 10 décembre 1988, p. D-4.

«Un américain à Tanger», Le Devoir, 14 janvier 1989, p. C-6.

«'amour des enfants», Possibles, vol. XIII, n 4, automne 1989, p. 11-16.

«Espace urbain et espace littéraire (en collaboration avec Jean-François Chassay), La Petite Revue de philosophie, vol. XI, no 1, automne 1989, p. 85-99.

«Abscisse et ordonnée de la vie», Cahiers de théâtre Jeu, n 50, 1989, p. 224-225.

«Lettres californiennes: céleste San Francisco » MTL, avril 1990, p. 43-47.

«Le Québec entre la culture et les cultures», Possibles, vol. XIV, nº 3, été 1990, p. 7-11 ${ }^{6}$.

«Marguerite Yourcenar: une femme libre dans le siècle», Le Devoir, 27 octobre 1990, p. D-1.

«Les combats de Maurice Nadeau», Le Devoir, $1^{\text {er }}$ décembre 1990, p. D-5.

«Que savez-vous de l’Estonie ?», Le Devoir, 2 février 1991, p. D-6.

«Lire m’affole», Liberté, vol. XXXIII, n 1 , février 1991, p. 50-55.

«Anne, Charlotte, Émily sauvées par le roman», Le Devoir, 16 mars 1991, p. D-1 et D-2.

«Carla entra» Le Devoir, 6 avril 1991, p. D-1 et D-2.

6. Éditorial et codirection du numéro avec Lise Gauvin. 
«Go alone», Liberté, vol. XXXIII, n² 2, avril 1991, p. 86-91.

«'écrivain et son public 》, Possibles, vol. XV, nº 4, automne 1991, p. 77-84.

«Simone de Beauvoir: la bourgeoise existentialiste», Le Devoir, 30 novembre 1991, p. D-1 et D-4.

«Monsieur Montréalais francophone», Autrement, «Série-monde- H.S. » n 62, mai 1992, p. 161-162.

«Résister à l'absurde», Liberté, vol. XXXIV, n 5, octobre 1992, p. 66-68.

«La mélancolie d'Hubert Aquin», Horizons philosophiques, vol. III, n 1, automne 1992, p. 31-41.

«Montréal inappropriable», Benoit Melançon et Pierre Popovic (dir.), Montréal 1642 1992 : le grand passage, Montréal, XYZ éditeur, coll. «Théorie et littérature»1994, p. 107-114.

«Power or "Unpower" of Fictional Subject: A Letter to Lise Gauvin in Reply to "What? You Too ?" », Barbara Godard (dir./tr.), Collaboration in the Feminine: Writings on Women and Culture from Tessera, Toronto, Second Story, 1994, p. 221-227.

«Le soin de la langue», Les Écrits, n 93, 1998, p. 83-947.

«La femme et la raison», Arcade, no 47, 1999, p. 17-22.

«L'écrivain est indispensable à la langue», Michel Plourde (dir.), avec la collaboration de Hélène Duval et de Pierre Georgeault, Le français au Québec, Conseil de la langue française, Les Publications du Québec, Gouvernement du Québec, Montréal, Fides, 2000, p. 422-423.

«Marco Polo, Rosa Luxembourg, et le Japon», Les Écrits, nº 103, décembre 2001, p. 4562.

«Actualité de Werther: Les souffrances du jeune Werther», Cahiers de théâtre Jeu, $\mathrm{n}^{\circ}$ 103, juin 2002, p. 143.

\section{I.7. Préfaces, présentation}

«Le sentiment du lieu », préface de Déjà l'agonie de Marco Mircone, Montréal, l'Hexagone, 1988, $81 \mathrm{p}$.

«La passion du double», préface pour Le fils d'Ariane de Micheline LaFrance, Montréal, Typo, 1996, $148 \mathrm{p}$.

«Le révélateur», texte de présentation inclus dans Les espaces du silence d'Éric Daudelin, Laval, Les 400 coups, 1998, non paginé ${ }^{8}$.

\section{I.8. Lettres journalistiques}

«L'arpenteur et le navigateur (suite) : la bonne foi et la déformation. À qui le Québec fait-il peur? Quelle littérature et quel Québec voulons-nous?», Le Devoir, 15 juillet 1997, p. A-7.

«Nos petits scandales», Le Devoir, 12 août 1997, p. A-6.

7. Discours de réception à l'Académie des Lettres du Québec.

8. Publié à l'occasion de l'exposition Les espaces du silence, à la Galerie Détour, Jambes (Belgique), en mars 1998. 
«Voulons-nous de la littérature ? Le droit au doute est au cœur de la liberté », Le Devoir, 13 août 1997, p. A-7.

«Le mot de l'académie», Le Devoir, 2 juillet 2002, p. A-1.

\section{PRIX ET DISTINCTIONS}

Prix du concours d'œuvres dramatiques radiophoniques de Radio-Canada, 1984, pour son texte L'enregistrement.

Grand Prix du livre de Montréal, 1990, pour Copies conformes.

Grand Prix Littéraire du Journal de Montréal, 1995, pour La démarche du crabe.

Bourse d'écriture du Fonds Gabrielle-Roy attribuée en 1996.

Membre de l'Académie des Lettres du Québec en 1998.

Prix du Gouverneur général, 2002, pour La gloire de Cassiodore.

\section{RÉCEPTION CRITIQUE ET ÉTUDES}

\section{III.1. Articles et chapitres de livres}

\section{III.1.1. Romans}

\section{III.1.1.1. Copies conformes}

L'HÉRAULT, Pierre, «Enquête sur l'identité : l'instance policière dans Copies conformes, La mauvaise foi et Paye-moi une bouffe, poète!», Tangence, n³ 38, décembre 1992, p. 8-23.

IRELAND, Susan, «Monique LaRue's Copies conformes: An Original Copy», Quebec Studies, n 15, automne 1992-hiver 1993, p. 21-30.

GOULD, Karen, «Copies conformes: la réécriture québécoise d'un polar américain », Études françaises, vol. XXIX, nº 1, printemps 1993, p. 25-35.

CHASSAY, Jean-François, «La contrainte américaine: Madeleine Monette et Monique LaRue», Benoit Melançon et Pierre Popovic (dir.), Montréal 1642-1992: le grand passage, Montréal, XYZ éditeur, coll. «Théorie et littérature» 1994, p. 219-229.

GUILLEMETTE, Lucie, «Le voyage et ses avatars dans Copies conformes de Monique LaRue: dérive et/ou délire identitaire», Canadian Issues, $n^{0} 16,1994$, p. 77-87.

SIMON, Sherry, «Les langues antagoniques de la ville», Le trafic des langues: traduction et culture dans la littérature québécoise, Montréal, Boréal, 1994, p. 128147.

CHASSAY, Jean-François, «L'invention d'une ville (Des villes et des fantômes) », L'ambiguïté américaine: le roman québécois face aux États-Unis, Montréal, XYZ éditeur, coll. «Théorie et littérature», 1995, p. 167-185.

IRELAND, Susan «American Stories by Monique LaRue and Jacques Godbout», Quebec Studies, n² 20, printemps-été 1995, p. 47-55.

ZUPANCIC, Metkna, «L'orphisme réécrit au féminin », Francophonie plurielle. Actes du congrès mondial du Conseil international d'études francophones tenu à Casablanca (Maroc), Ginette Adamson et Jean-Marc Gouanvic (dir.), Québec, HMH Hurtubise, 1995, p. 51-63.

CANTY, Daniel, «Le jeu d'imitation dans Copies conformes de Monique LaRue», Voix et Images, no 62, hiver 1996, p. 324-336. 
GOULD, Karen, «Rewriting "America": Violence, Postmodernity and Parody in the Fiction of Madeleine Monette, Nicole Brossard and Monique LaRue», Mary Jean Green, Karen Gould, Micheline Rice Maximin, Keith L. Walker, Jack A. Yeager (dir.), Postcolonial Subjects: Francophone Women Writers, Minneapolis, University of Minnesota, n 22, 1996, p. 186-209.

SOLBERG, Janet, Quant à moi. Témoignages des Français et des francophones, «Text Bank», Boston, Heinle \& Heinle, 1996, p. 86-889.

DION, Robert, «La littérature (im)médiate», Le moment critique de la fiction, Québec, Nuit blanche éditeur, 1997, p. 39-59.

SAINT-GELAIS, Richard, «L'Amérique virtuelle de la science fiction québécoise» Jaap Lintvelt, Richard Saint-Gelais, Will Verhoven et Catherine Raffi-Béroud (dir.), Roman contemporain et identité culturelle en Amérique du Nord/Contemporary Fiction and Cultural Identity in North America, Québec, Nota bene, 1998, p. 141153.

BOIVIN, Aurélien, «Copies conformes ou le piratage électronique», Québec français, $\mathrm{n}^{0}$ 116, hiver 2000, p. 92-94.

DION, Robert, «"Let's Talk English Here”. Les représentations de l'anglais dans Copies conformes et Volkswagen blues», Barbara Buchenau et Annette Paatz (dir.), Do the Americas Have a Common Literary History?, Francfort/Berlin/Berne/Bruxelles/ NewYork/Oxford/Vienne, Peter Lang, coll. «Interamericana », 2002, p. 427-447.

\section{III.1.1.2. La démarche du crabe}

GUILLEMETTE, Lucie, «À la croisée du hasard et du déterminisme: atomisme logique et fractalité postmoderne dans La démarche du crabe de Monique LaRue», Tangence, no 61, décembre 1999, p. 113-146.

\section{III.1.2. Dramatique à la radio}

\section{III.1.2.1. L'enregistrement}

LAMY, Suzanne, «Au contact d'une voix», Revue francophone de Louisiane, vol. I, n 1 , printemps 1986, p. 16-27.

\section{III.1.3. Collectif}

\section{III.1.3.1. Plages}

FISHER, Claudine G., «Sensibilités française et québécoise dans Plages», Revue francophone de Lafayette, vol. V, nº 1, printemps 1990, p. 64-70.

\section{III.1.4. Autres}

GOULD, Karen, "Refiguring the Mother: Quebec Women Writers in the 80s», International Journal of Canadian Studies/Revue internationale d'études canadiennes, no 6, automne 1992, p. 113-125.

SAINT-MARTIN, Lori, «Le métaféminisme et la nouvelle prose féminine au Québec», Voix et Images, n 52, automne 1992, p. 78-88.

9. Document pédagogique sur un extrait de Copies conformes. 
SAINT-MARTIN, Lori, «Le corps et la fiction à réinventer: métamorphoses de la maternité dans l'écriture des femmes au Québec», Recherches féministes, vol. 7, $\mathrm{n}^{\circ} 2$, 1994, p. 115-134.

ZUPANCIC, Metkna, «Feministicna proza: miti in utopija: Margaret Atwood, Chantal Chawaf, Hélène Cixous, Madeleine Monette, Monique LaRue, Berta Bojetu », Primerjalna-Knjizevnost, vol. XVIII, no 2, 1995, p. 1-16.

VAN”T LAND, Hilligje, «La Kérouacquisition du territoire américain : Poulin, Godbout et LaRue comme représentants du discours littéraire québécois des années 80 », Rapports. Het Franse boek, vol. XXVI, nº 22, 1996, p. 31-40.

IRELAND, Susan, "The Figure of the Mother in the Novels of Monique LaRue», Roseanna Lewis Dufault (dir.), Women by Women: The Treatment of Female Characters by Women Writers of Fiction in Quebec since 1980, Canbury, Associated UP, 1997, p. 34-53.

MACLURE, Jocelyn, «Authenticités québécoises: le Québec et la fragmentation contemporaine de l'identité », Globe. Revue internationale d'études québécoises, vol. I, n ${ }^{\circ}$ 1, 1998, p. 9-35.

GUILLEMETTE, Lucie, «Littérature québécoise et expérience continentale: américanité et/ou américanisation?», L'Action nationale, vol. XC, n 6, juin 2000, p. 51-65.

SAWICKA, Sylwia, «Le poids de l'américanité dans les romans de Monique LaRue et Jacques Godbout», Regards sur la littérature québécoise. Hommage à Gaston Miron, Actes du troisième colloque des jeunes chercheurs européens en littérature québécoise, Rome, Bulzoni Editore, 2001, p. 141-155.

\section{III.2. Comptes rendus et articles de presse}

\section{III.2.1. Romans}

\section{III.2.1.1. La cohorte fictive}

OUELLETTE-MICHALSKA, Madeleine, «Neuf et vibrant», Châtelaine, vol. XX, n 12 , décembre 1979, p. 26.

BEAUSOLEIL, Claude, «Entre maternité et écriture», Spirale, nº 8, janvier 1980, p. 10.

MARTEL, Réginald, «Mort d'un ouvrier», La Presse, 5 janvier 1980, p. B-3.

ROYER, Jean, «Monique LaRue: un premier roman réussi», Le Devoir, 26 janvier 1980, p. 19.

OUELLETTE-MICHALSKA, Madeleine, «Un bilan littéraire», Spirale, nº 7, mars 1980, p. 12-13.

ROY, Michèle, «La cohorte fictive», Le bulletin Pantoute, juin-juillet-août 1980, p. 7.

BRAUTMAN, Davida, «LaRue, Monique: La cohorte fictive», The French Review, vol. LIV, nº 3, février 1981, p. 506.

GRÉGOIRE, Claude, «La cohorte fictive par Monique Larue» Gilles Dorion (dir.), avec la collaboration de Aurélien Boivin, de Roger Chamberland et de Gilles Girard, Dictionnaire des œuvres littéraires du Québec, tome VI, 1976-1980, Montréal, Fides, 1994, p. 158. 


\section{III.2.1.2. Les faux fuyants}

MARTEL, Réginald, «Les faux fuyants: un regard de glace sur la prison humaine» La Presse, 22 mai 1982, p. C-3.

OUELLETTE-MICHALSKA, Madeleine, «Monique LaRue : où se trame le réel», Le Devoir, 29 mai 1982, p. 19.

ROYER, Jean, «Monique LaRue : le corps des mots», Le Devoir, 26 juin 1982, p. 13.

CHARTIER, Monique, «Notre choix, Les faux fuyants de Monique LaRue - Le livre du mois choisi par le comité de rédaction - Monique LaRue répond à nos questions», Nos livres, vol. XII, août-septembre 1982, p. 5-7, cf. \# 343.

LEBLOND, Chantal, «Des Faux fuyants fragiles» Le Continuum, 1982, p. 10.

MAILHOT, Michèle, «Les faux fuyants», Le Droit, 25 septembre 1982, p. 14.

MARCOTTE, Gilles, «Des personnages guère doués pour l'amour», L'Actualité, vol. VII, no 11, novembre 1982, p. 129.

LÉVESQUE, Gaëtan, «Regards lucides sur la société», Voix et Images, vol. VIII, n 1 , automne 1982, p. 155-156.

MAILHOT, Michèle, «Les Faux fuyants», Lettres québécoises, n² 27, automne 1982, p. 2022.

LAFOREST, Marty, «Monique LaRue: Les faux fuyants», Livres et auteurs québécois 1982. Revue critique de l'année littéraire, Québec, Les Presses de l’Université Laval, 1983, p. 63-64.

\section{III.2.1.3. Copies conformes}

ROYER, Jean, «Adieu femmes romanesques: Copies conformes», Le Devoir, 18 novembre 1989, p. D-8.

FORTIN, Marie-Claude, «Le vol du Faucon», Voir, 23 novembre 1989, p. 21.

CLOUTIER, Guy, «Avec Copies conformes Monique LaRue joint son nom aux plus grands écrivains d'ici», Le Soleil, 25 novembre 1989, p. F-11.

BASILE, Jean, «Amour, micro-ordinateur et suspense à San Francisco », La Presse, 2 décembre 1989, p. K-1.

MORISSETTE, Rodolphe, «LaRue : l'amoureuse intelligente», Journal de Montréal, 2 décembre 1989, p. We-7.

DEMOUZON, Alain, «Bien cher monsieur Hammett», Le Monde, 29 décembre 1989, p. 34.

RIOUX, Hélène, «Mystère au programme», Journal d'Outremont, décembre 1989, p. 19.

ALIZET, Jean-Claude, «Monique LaRue: Copies conformes» «Sueurs froides» l'année du polar, de la «SF», du fantastique et de l'espionnage, Amiens, Encrage, 1990, p. 185.

BIRON, Michel, «L'origine et l'original», Spirale, n 94, février 1990, p. 10-11. 
FORTIN, Marie-Claude, «Frissons à Frisco 》, Montréal, février 1990, p. 53.

MARCOTTE, Gilles, «Fragments d'une civilisation» L'Actualité, vol. XV, $\mathrm{n}^{0}$ 2, février 1990, p. 117.

VOISARD, Anne-Marie, «Pour Monique LaRue, l'écriture sert d'oxygène», Le Soleil, 10 février 1990, p. D-9.

CLOUTIER, Guy, «Silicon Valley», Le Magazine littéraire, n² 275, mars 1990, p. 75.

GAUDREAULT, André, «Une femme interroge son choix de vie» Le Nouvelliste, 10 mars 1990, p. A-12.

PICARD, Geneviève, «Les femmes et le roman policier», Elle-Québec, juin 1990, p. 3840.

SABOURIN, Claude, «Les espaces du faux», Lettres québécoises, nº 57, printemps 1990, p. 52.

YERGEAU, Normand, «Copies conformes», Le Magazine affaires plus, juillet-août 1990, p. 56.

CÔTÉ, Lucie, «Une promenade littéraire à San Francisco » La Presse, 4 août 1990, p. I-3.

BEAUDOIN, Réjean, «Détecteurs de mensonges» Liberté, vol. XXXII, n 6, décembre 1990, p. 94-100.

SUTHERLAND, Ronald, «Two Quebec Novels Short and Sharp», The Globe and Mail, 4 janvier 1997, p. D-11.

\section{III.2.1.4. La démarche du crabe}

CAYOUETTE, Pierre, «Une erreur de désir», Le Devoir, 9 avril 1995, p. D-1.

VOISARD, Anne-Marie, «Changement de carapace» Le Soleil, 15 avril 1995, p. C-7.

CREVIER, Gilles, «Le commencement de la fin d'un homme à la dérive», Journal de Montréal, 22 avril 1995, p. We-16.

POULIN, Andrée, Jean-Claude SURPRENANT et Marie-Paule VILLENEUVE, «La démarche du crabe», Le Droit, 22 avril 1995, p. A-10.

MARTEL, Réginald, «Un être venu de partout et de nulle part, irrémédiablement condamné à y retourner», La Presse, 23 avril 1995, p. B-3.

ALARD, Jacques, «Voyage au cœur de la vie», Le Devoir, 29 avril 1995, p. D-5.

FORTIN, Marie-Claude, «Le passé recomposé» Voir, avril 1995, p. 9.

FRANCOEUR, Martin, «Le poids des souvenirs d'un homme en détresse», Le Nouvelliste, 3 juin 1995, p. 6.

CHAREST, Rémi, «Successful Forays Across Gender Lines», The Gazette, 10 juin 1995, p. I-5.

MARTEL, Réginald, «Monique LaRue : l'idée, l'émotion, l'intrigue et le reste» La Presse, 11 juin 1995, p. B-3.

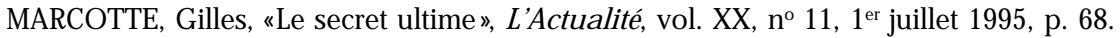

JOUBERT, Lucie, «Trilogie des corps recyclés», Spirale, n 144, septembre-octobre 1995, p. 9.

BORDELEAU, Francine, «Le choc du passé», Lettres québécoises, n 79, automne 1995, p. 19. 
CHAMBERLAND, Roger, «Monique LaRue: La démarche du crabe» Québec français, no 99, automne 1995, p. 20.

BEAUMIER, Jean-Paul, «Monique LaRue: La démarche du crabe», Nuit blanche, $\mathrm{n}^{\circ} 62$, hiver 1995-1996, p. 18-19.

NICOL, Patrick, «Book of the Year», Encyclopaedia Britannica, «Literature: French Canada», 1996, p. 232.

\section{III.2.1.5. La gloire de Cassiodore}

CHARTRAND, Robert, «En classe et ailleurs», Le Devoir, 19 janvier 2002, p. D-3.

GUAY, Hervé, «Quel héritage? Monique LaRue» Le Devoir, 19 janvier 2002, p. D-1.

FORTIN, Marie-Claude, «Monique LaRue : à bonne école» Voir, 24 janvier 2002, p. 27.

APOSTOLSKA, Aline, «Gloire et misère des cégeps», La Presse, 27 janvier 2002, p. B-3.

MARTEL, Réginald, «Un regard pénétrant sur le monde de l'éducation» La Presse, 27 janvier 2002, p. B-3.

BOURGAULT-CÔTÉ, Guillaume, «Colère de prof», Le Soleil, 3 février 2002, p. B-5.

VILLENEUVE, Marie-Paule, «Le cégep en question» Le Droit, 9 février 2002, p. A-17.

MONTESSUIT, Carmen, «Tout ce qui se passe dans un collège... », Journal de Montréal, 17 février 2002, p. 46.

PAQUIN, Caroline, «Entre écrivain et enseignant», Impact campus, vol. XVI, n ${ }^{\circ} 19$, 19 février 2002.

CRÉPEAU, Jean-François, «Le Cégep heureux n'a pas d'histoire: croyance populaire!», Le Canada français, 20 mars 2002, p. C-8.

BOUCHARD, Marie-Pierre, «Apologie de l'intelligence», Accès Laurentides, 12 avril 2002, p. 14.

FORTIN, Marie-Claude, «Bons bulletins», Clin d'œil, avril 2002, p. 30.

LAURIN, Danielle, «Du cégep à l'université», Elle-Québec, avril 2002, p. 66.

ROY, Monique, «Vie de prof», Châtelaine, mai 2002, p. 36.

DOUCET, Sophie, «Dans les couloirs», Femme, mai-juin 2002, p. 10.

GAUDREAU, Hélène, «Défense d'un humanisme actuel: La gloire de Cassiodore de Monique LaRue», Nuit blanche, no 87, été 2002, p. 28-29.

BROCHU, André, «Cassiodore à l'heure d'Internet», Lettres québécoises, n 107 , automne 2002, p. 19.

\section{III.2.2. Dramatique à la radio}

\section{III.2.2.1. L'enregistrement}

LAPOINTE, Gilles, «'empreinte de la voix », Spirale, n 48, décembre 1984, p. 23.

\section{III.2.3. Collectif}

\section{III.2.3.1. Plages}

GAMACHE, Chantal, «Des regards qui s'attardent», Le Devoir, 4 avril 1987, p. D-3. 


\section{III.2.4. Essais}

\section{III.2.4.1. Promenades littéraires dans Montréal}

MATTEAU, Hélène, «Demain, mon Réal m’attend» Le Québec littéraire, n 3, été 1989, p. 22-33.

LEPAGE, Jocelyne, «Le Montréal des écrivains: plus vrai que nature mais amputé de moitié », La Presse, 2 décembre 1989, p. K-2.

BOIVIN, Jean-Roch, «La ville est un roman» Voir, 7 décembre 1989, p. 21.

BOIS, Anne, «From Montréal's Streets to France's Forests», The Gazette, 20 janvier 1990, p. K-8.

RIOUX, Hélène, «Lire et marcher: un coup double», Journal d'Outremont, janvier 1990, p. 17.

LÉGER, Hugo, «Montréal à la page» MTL, février 1990, p. 6.

POISSON, Roch, «Les mille visages d'un grand village», Le Bel Âge, avril 1990, p. 8.

LANGEVIN, Lysanne, «Le droit de cité/citer», Arcade, n 19, printemps 1990, p. 86-90.

JONASSAINT, Jean, «Un double hommage à notre littérature», Lettres québécoises, $n^{\circ} 58$, été 1990, p. 46-47.

\section{III.2.4.2. L'arpenteur et le navigateur ${ }^{10}$}

SROKA, Ghila B., «De LaRue à la poubelle», Tribune juive, vol. XIV, nº 3, mars 1997, p. 4-5.

MARTEL, Réginald, «À propos de Ghila B. Sroka et Monique LaRue», La Presse, 15 avril 1997, p. C-13.

SALETTI, Robert, «Être du pays de ses enfants» Le Devoir, 19 avril 1997, p. D-6.

BISSO NNETTE, Lise, «Un cauchemar», Le Devoir, 26 avril 1997, p. B-3.

NEPVEU, Pierre, «Recension de L'arpenteur et le navigateur dans Tribune juive. Lire à l'envers: Ghila Sroka a fait une lecture aberrante et grossière de l'ouvrage de Monique LaRue», Le Devoir, 26 avril 1997, p. A-11.

ALAVO, Yves, «Reconnaître les pionniers», Tribune juive, vol. XIV, nº 4, mai 1997, p. $24-25$.

BILARD, Jean-Antonin, «L'endroit de l'envers ou de haut en bas. Lettre ouverte à Ghila B. Sroka, fondatrice et directrice de Tribune juive et de La Parole métèque et mon amie pure soie préférée», Tribune juive, vol. XIV, n 4 , mai 1997, p. 22-23.

CHARLER, Ghislaine, «Plaidoyer pour un Québec ouvert», Tribune juive, vol. XIV, $\mathrm{n}^{\circ} 4$, mai 1997, p. 21.

DE SĖVE, Micheline, «L'arpenteur et le navigateur: malaise au pluriel», Le Devoir, 5 mai 1997, p. A-7; Tribune juive, vol. XIV, n 4, mai 1997, p. 16.

NAVARRO, Pascale, «Littérature nationale : l'imaginaire souverain», Voir, 15 mai 1997, p. 10-11.

10. Plusieurs textes rassemblés en III.2.4.2. ne portent pas directement sur L'arpenteur et le navigateur mais bien sur ce qu'on s'est empressé d'appeler «'affaire LaRue», voulant désigner ainsi la polémique autour de l'identité québécoise que ce petit livre a suscitée. 
DEMERS, Jeanne, «L'arpenteur et le navigateur: une lecture erronée et destructive» Le Devoir, 17 mai 1997, p. A-9.

HÉBERT, François, «Douces censures», Le Devoir, 21 mai 1997, p. A-7.

KLANG, Gary, «Lettre ouverte à madame Monique LaRue», Tribune juive, vol. XIV, $n^{\circ} 4$, mai 1997, p. 20.

NEPVEU, Pierre, «Monique LaRue et l'avenir de la littérature québécoise», Tribune juive, vol. XIV, nº 4, mai 1997, p. 14.

SROKA, Ghila B., «La fin des illusions » et «La seule grossièreté que je connaisse est la lâcheté », Tribune juive, vol. XIV, nº 4, mai 1997, p. 4-12.

YANACOPOULO, Andrée, «On n'emprisonne plus Voltaire», Tribune juive, vol. XIV, no 4, mai 1997, p. 19.

NEPVEU, Pierre, «Notes sur un angélisme au pluriel», Le Devoir, 9 juin 1997, p. A-7.

NEPVEU, Pierre, «L'impossible oubli», Le Devoir, 10 juin 1997, p. A-9.

ANGENOT, Marc, «Littérature et nationalisme», Tribune juive, vol. XIV, nº 5, juin-juillet 1997, p. 12-16.

HORIC, Alain, «Lettre ouverte à $\mathrm{M}^{\mathrm{me}}$ Jeanne Demers», Tribune juive, vol. XIV, $\mathrm{n}^{0} 5$, juinjuillet 1997, p. 22.

KLANG, Gary, «Trop c'est trop : peut-on chanter le pays accueillant sans sortir les grandes orgues du nationalisme? Lettre ouverte à Pierre Nepveu», Tribune juive, vol. XIV, no 5, juin-juillet 1997, p. 18-20; Le Devoir, 3 juillet 1997, p. A-7.

MACKLOVITCH, Lison, «Une honte», La Voix sépharade, juin-juillet 1997, p. 6.

TRUQUE, Yvonne-América, «To Add to the Confusion de l'Affaire LaRue», Tribune juive, vol. XIV, no 5, juin-juillet 1997, p. 16-17.

WOLF, Marc-Alain, «Récit d'une calomnie» La Voix sépharade, juin-juillet 1997, p. 6.

LÉVESQUE, Claude, «À propos du “Québec xénophobe"», Le Devoir, 3 juillet 1997, p. A-7.

JASMIN, Claude, «À qui le Québec fait-il peur?», Le Devoir, 15 juillet 1997, p. A-7.

CORNELLER, Louis, «Le blues du tabarnacos», Le Devoir, 16 juillet 1997, p. A-7.

KLANG, Gary, «Bas les masques», Le Devoir, 28 juillet 1997, p. A-7.

SROKA, Ghila B., «Pourquoi ce numéro spécial ?» «La réconciliation» «Mise au point», «'accuse Pierre Nepveu», «Lettre ouverte à Claude Jasmin», «Lettre ouverte à Emille Olivier», «Lettre ouverte à l'UNEQ », La Parole métèque, nº 30, juillet 1997, p. 4-6, 8, 12, 15-16.

FOGLIA, Pierre, «J'aime pas quand tu chantes», La Presse, 26 août 1997, p. A-5.

HOMEL, David, «War on Words Fought on Familiar Turf», The Gazette, 6 septembre 1997, p. K-5.

DAVIES, Robert, «Hasn't a Racist Bone in Her Body», The Gazette, 24 septembre 1997, p. B-2.

LAFLĖCHE, Guy, «Le racisme de L'arpenteur et le navigateur par Monique LaRue», Tribune juive, vol. XV, nº 5, juin-juillet 1998, p. 18-20. 


\section{ENTRETIENS ET PORTRAITS}

\section{IV.1. Revues et journaux}

ROYER, Jean, «Le corps des mots», Écrivains contemporains. Entretiens 3, Montréal, l'Hexagone, 1982, p. 314-320.

CHASSAY, Jean-François, «Québec 86: Monique LaRue», Magazine littéraire, n 234, octobre 1986, p. 105-106.

JARQUE, Alexandra, «Monique LaRue: la vie n'est pas un roman », Nuit blanche, vol. XVIII, mars-avril-mai 1991, p. 10-13 (repris dans le numéro spécial 15e anniversaire, vol. LXIX, hiver 1997, p. 66-69).

HALEY, Achmy, «Pourquoi écrivez-vous?», La Presse, 19 novembre 1995, p. B-6.

HALLEY, Achmy, «D’après-vous, l'écriture a-t-elle un sexe?» La Presse, 3 mars 1996, p. B-4.

ROYER, Jean, «Monique LaRue et Jean-François Chassay: l'écrivain et l'esprit des lieux », Écrivains contemporains: nouveaux entretiens, Montréal, Trait d'union, 1999, p. 17-20.

MARSAN, Jean-Sébastien, «Un nivellement par le bas», La Force des mots, CSN, n 9, mars 2002, p. 2-4. 